\title{
Injection of local anesthetic lateral to vertebral transverse process instead of conventional method for interscalene block in difficult cases with poor view
}

\author{
Reza Heidarifar ${ }^{1}$, Seyyed Mohammadreza Amini ${ }^{1}$, Sarah Lotfi ${ }^{2}$, Abbas Ahmadi ${ }^{1}$ \\ Author affiliation: \\ 1- Department of Anesthesiology, School of Medicine, Shahid Beheshti Hospital, Qom University of Medical Sciences, Qom, \\ Iran. \\ 2- Gynecology Oncology Department of Obstetrics and Gynecology, School of Medicine Nekouei-Hedayati-Forghani Hospital \\ Qom University of Medical Sciences, Qom, Iran.
}

Correspondence: Abbas Ahmadi; E-mail: abbasAhmadi27@yahoo.com

Key words: Nerve Block/methods; Anesthetics, Local; Peripheral Nerves / diagnostic imaging; Ultrasonography, Interventional / methods

Citation: Heidarifar R, Amini SM, Lotfi S, Ahmadi A. Injection of local anesthetic lateral to vertebral transverse process instead of conventional method for interscalene block in difficult cases with poor view. Anaesth. pain intensive care 2021;26(1):126-127. DOI: $10.35975 / a p i c . v 26 i 1.1783$

Ultrasound guided (USG) peripheral nerve block techniques have become increasingly popular in regional anesthesia in the recent few years. USG interscalene brachial plexus block provides effective analgesia after a variety of of surgeries and have been shown to reduce opioid consumption, decrease postoperative nausea and vomiting (PONV), improve patient satisfaction, and decrease recovery room length of stay. ${ }^{1}$ Regardless of all of these advantages, there have been some complications associated with this technique. The most important complication is the phrenic nerve block. Therefore, impaired respiratory function is considered a relative contraindication to interscalene plexus block. It has been shown to cause ipsilateral hemidiaphragmatic paresis almost always due to phrenic nerve block. ${ }^{2,3}$

With the introduction of the USG in the regional anesthesia, it is now relatively easy to target any desired portion of the brachial plexus and achieve surgical anesthesia and analgesia for the entire upper extremity. Moreover, the duration of the regional anesthesia can be extended by inserting a catheter and continuous infusion of local anesthetics (LA) through it. However, in some cases it may be difficult to attain good view for a correct block. ${ }^{4}$ There is a need to identify the optimal location for LA administration during an interscalene block. The injection site chosen for any type of block can affect its quality, time to onset, and the duration. Interscalene plexus block is performed at the C5 level. In conventional method the US transducer is placed transversally on the

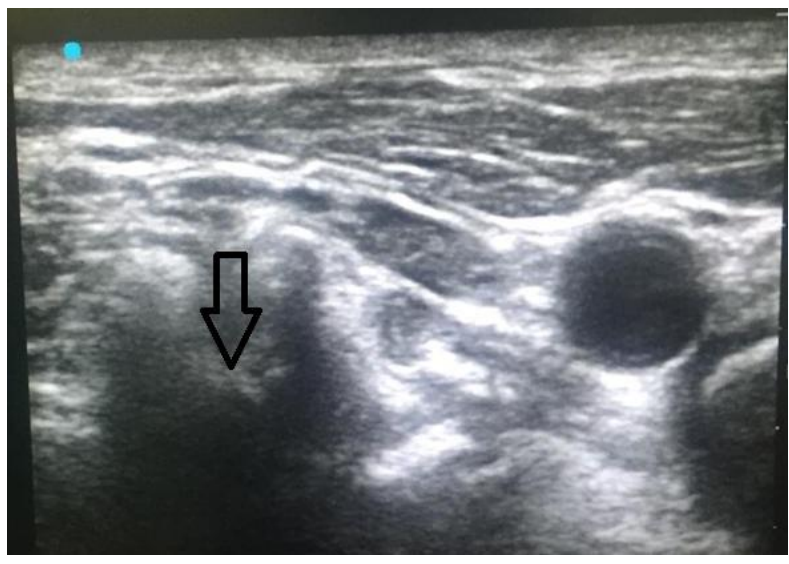

Figure 1: Shows the spread of the LA solution (arrow)

neck at the level of the superior pole of the thyroid cartilage and then slightly aligned laterally, where the nerve roots are observed between the anterior and middle scalene muscles at the interscalene groove. The needle insertion site is determined under US guidance as the point where the posterolateral border of the sternocleidomastoid muscle starts. The final target position of the needle is immediately posterior to the space between the $\mathrm{C} 5$ and $\mathrm{C} 6$ roots.

The needle is inserted laterally at the posterior border of the sternocleidomastoid muscle and advanced under US guidance using the in-plane technique to reach to the 
brachial plexus. LA drug is administered under real-time visualization of the needle tip and the distribution of the solution. ${ }^{5}$

By this capability we would decrease the block failure and improve block efficacy. More clinical trials are needed to ascertain the accuracy and the comparative benefits of this new technique.

\section{References}

1. Harbell MW, Kolodzie K, Behrends M, Ma CB, Kinjo S, Yap E, et al. Extraplexus versus intraplexus ultrasound-guided interscalene brachial plexus block for ambulatory arthroscopic shoulder surgery: A randomized controlled trial. PloS one. 2021:16(2):e0246792. [PubMed] DOI: 10.1371/journal.pone.0246792

2. El-Boghdadly K, Chin KJ, Chan VW. Phrenic nerve palsy and regional anesthesia for shoulder surgery: anatomical, physiologic, and clinical considerations. Anesthesiology. 2017;127(1):173-91. 10.1097/ALN.0000000000001668

3. Schwenk ES, Gandhi K, Viscusi ER. Perioperative management of Interscalene block in patients with lung disease. Case Rep Anesthesiol. 2013;2013:986386. PubMed] DOI: 10.1155/2013/986386

4. Madison SJ, Humsi J, Loland VJ, Suresh PJ, Sandhu NS, Bishop $M J$, et al. Ultrasound-guided root/trunk (interscalene) block for hand and forearm anesthesia. Reg Anesth Pain Med. 2013 MayJun;38(3):226-32. [PubMed]

DOl: 10.1097/AAP.0b013e3182890d50

5. Balaban O, Dülgeroğlu TC, Aydın T. Ultrasound-guided combined interscalene-cervical plexus block for surgical anesthesia in clavicular fractures: a retrospective observational study. Anesthesiol Res Pract. 2018 Jun 3;2018:7842128. [PubMed] DOI: 10.1155/2018/7842128 